Proceedings - 20th Annual International Conference - IEEE/EMBS Oct. 29 - Nov. 1, 1998, Hong Kong

\title{
MULTIRESOLUTION WAVELET ANALYSIS OF HEART RATE VARIABILITY FOR HEART-FAILURE AND HEART-TRANSPLANT PATIENTS
}

\author{
Malvin C. Teich, Fellow, IEEE \\ Departments of Electrical \& Computer Engineering, \\ Biomedical Engineering, and Physics \\ Boston University \\ 8 Saint Mary's Street, Boston, Massachusetts 02215, USA \\ e-mail: teich@bu.edu \\ URL: http://ece.bu.edu/ECE/faculty/homepages/teich.html/
}

\begin{abstract}
We have carried out a study on a collection of electrocardiograms from patients who suffer from congestive heart failure, heart-transplant patients, and normal subjects, using wavelet-based multiresolution techniques and receiver-operating-characteristic (ROC) analysis. The scale-dependent wavelet-coefficient standard deviation $\sigma_{\text {wav }}(m)$ is found to be superior to two commonly used heart-rate-variability measures for diagnosing cardiac dysfunction, the interbeat-interval standard deviation $\sigma_{\text {int }}$ and the spectral scaling exponent $\delta$. A recent IsraeliDanish study of diabetic patients that confirms our observations is discussed.
\end{abstract}

\section{INTRODUCTION}

The interbeat-interval (R-R) time series of the human heart exhibits scaling behavior, as evidenced by the powerlaw form of its spectrum, which decreases as $f^{-\delta}$ for sufficiently low frequencies $f[1,2]$. However other features, associated with physiological markers, are also present in the power spectrum at particular frequencies [3]. Moreover, it is well known that the heartbeat time series is nonstationary, reflecting biological adaptability.

Multiresolution wavelet analysis $[4,5,6,7,8]$ provides an ideal means of decomposing a signal into its components at different scales, and at the same time has the salutary effect of eliminating nonstationarities $[9,10,11]$. We carried out a study [12] in which wavelets were used to analyze the sequence of interbeat intervals from a standard electrocardiogram (ECG) database [13], and discov-ered a critical scale window over which it was possible to perfectly discriminate heart-failure patients from normal subjects. The presence of this scale window has been confirmed in a recent Israeli-Danish study of diabetic patients who had not yet developed clinical signs of cardiovascular disease [14]. These two studies [12, 14], in conjunction with our earlier investigations involving the counting statistics of the heartbeat $[15,16,17]$ (as opposed to the time-interval statistics considered here), lead to the conclusion that scale-dependent measures (such as the wavelet-coefficient standard deviation) outperform scaleindependent ones (such as the scaling exponent $\delta$ ) in discriminating patients with cardiac dysfunction from normal subjects. It should be pointed out, nevertheless, that wavelet analysis provides an excellent technique for estimating the scaling exponent.

\section{METHODS}

The multiresolution-analysis procedure consists of transforming the discrete-time sequence of R-R intervals $s=$ $\left\{\tau_{i}\right\}$ into a space of wavelet coefficients. The transformed signal can be thought of in terms of a landscape over a two-dimensional plane whose axes are interbeat-interval number $i$ and scale $m$. Smaller scales correspond to more rapid variations and therefore to higher frequencies. The height is the value of the corresponding wavelet coefficient. With such a three-dimensional construct it is possible to simultaneously trace the behavior of the R-R sequence at multiple scales, as it proceeds in time.

Technically the coefficients are obtained by carrying out the discrete wavelet transform $[4,7,12]$

$$
W_{m, n}^{\mathrm{wav}}(s)=2^{-m / 2} \sum_{i=0}^{M-1} \tau_{i} \psi\left(2^{-m} i-n\right)
$$

where the scale variable $m$ and the translation variable $n$ are nonnegative integers, and $M$ represents the total number of $\mathrm{R}-\mathrm{R}$ intervals analyzed. The discrete wavelet transform is evaluated at the points $(m, n)$ in the scaleinterval-number plane. Because certain wavelets $\psi$ have 
vanishing moments, polynomial trends in the signal are automatically eliminated by the process of wavelet transformation $[9,10,11]$.

An example of applying multiresolution analysis to an interbeat-interval time series, using the Haar wavelet, is provided in Fig. 1. The left panel shows the original wavelet, a function which is, by definition, $\psi(x)=1$ for $x=0, \ldots, 0.5 ; \psi(x)=-1$ for $x=0.5, \ldots, 1$; and $\psi(x)=0$ elsewhere. The middle panel shows an example of the wavelet scaled by a factor of $m=4$, which makes is last for 16 samples rather than 1 , and delayed by a factor of $n=3$ times the length of the wavelet (hence it's beginning at $x=48$ ). In the right panel, the signal is multiplied by the wavelet, then all remaining points (i.e. those between 48 and 63 in this example) are added, and the result is the wavelet coefficient for the time $i=48$ (beginning of the wavelet) and the scale $m=4$. This is tantamount to adding the eight $R R$ intervals between intervals 48 and 55 inclusive, and then subtracting the eight subsequent $R R$ intervals between intervals 56 and 63, inclusive. Moving - this window across time allows us to see how the coefficient changes according to time, and varying the scale of the window yields finer or coarser resolution. We have previously shown that results for the wavelet coefficients are reasonably independent of the form of the wavelet function; we use Daubechies 10-tap wavelets [4] here.

Since the signal $s$ fluctuates in time, so too does the sequence of wavelet coefficients at any given scale, though their mean is zero. A natural measure for this variability is the wavelet-coefficient standard deviation, as a function of scale:

$$
\sigma_{\text {wav }}(m)=\left[\frac{1}{N-1} \sum_{n=0}^{N-1}\left(W_{m, n}^{\text {wav }}(s)-\left\langle W_{m, n}^{\text {wav }}(s)\right\rangle\right)^{2}\right]^{\frac{1}{2}},
$$

where $N$ is the number of wavelet coefficients at a given scale $m\left[N=\operatorname{int}\left(M / 2^{m}\right)\right][12]$.

\section{RESULTS}

We have established [12] that, at scales $m=4$ and 5 , $\sigma_{\text {wav }}(m)$ completely separates 15 heart-failure patients from 12 normal subjects in a standard ECG database [13]; despite the presence of atrial fibrillation in three of the heart-failure patients. As shown in the left panel of Fig. 2, this scale window lies between $2^{4}-2^{5}=16-32$ heartbeat intervals (roughly $0.2-0.5 \mathrm{~min}$ ).

The depression of the wavelet-coefficient standard deviation for the heart-failure patients, as well as for a sudden cardiac death (SCD) patient, at these particular scales is likely associated with the impairment of autonomic ner-vous system function. Baroreflex modulations of the sympathetic or parasympathetic tone typically lie in the range $0.04-0.09 \mathrm{~Hz}(0.2-0.5 \mathrm{~min})$, which corresponds to the time window where $\sigma_{\text {wav }}(m)$ is suppressed.
The most severe depression of the wavelet-coefficient standard deviation is exhibited by heart-transplant patients, for whom innervation by the autonomic nervous system is reduced, as is evident in the bottom-most curve in Fig. 2. Whether re-innervation ultimately ameliorates this suppression is, as yet, unknown.

This window of separation has also been observed by Ashkenazy et al. [14], as shown in the right panel of Fig. 2. Their data set comprised 10 diabetic patients who were otherwise healthy, one patient who had suffered a myocardial infarction, and one heart-transplant patient. In all cases, including the heart-transplant patient, they found that the same range of scales is useful in distinguishing normal subjects and patients with cardiac dysfunction. In their analysis of 12 patients and 21 normal subjects, only one normal standard-deviation curve fell among those of the patients.

The perfect separation between the heart-failure patients and normal subjects observed in our study endorses the choice of $\sigma_{\text {wav }}(m)$ as a useful measure. The results of most studies are seldom so clear-cut, however. In circumstances where there is incomplete separation between two classes of subjects, as observed for other measures using these identical data sets $[18,19]$, or in applying our measure to large collections of out-of-sample data sets, the relative abilities of different measures for determining the presence of disease is best established by the use of receiver-operating-characteristic (ROC) analysis [20].

Overlap between the two classes of subjects can also be increased by reducing the durations of the ECG recordings. We recently used ROC analysis to quantitatively compare the tradeoff between data length and discriminability provided by $\sigma_{\text {wav }}(m)$ and by two other widely used heart-rate-variability measures of cardiac dysfunction, the interbeat-interval standard deviation $\sigma_{\text {int }}$ and the spectral scaling exponent $\delta$ [21]. Moreover, we developed a mathematical model for heartbeat time-series generation for both heart-failure patients and normal subjects [21]. We discuss these two analyses in turn.

\section{ROC ANALYSIS}

ROC analysis is a useful and general method for establishing the tradeoff between reduced data length on the one hand, and misidentifications (misses and false positives) on the other. The ROC curve is a plot of sensitivity $v s$ specificity as the threshold parameter is swept. Unlike the determination of the $p$-value for statistical significance, ROC analysis relies on no implicit assumptions about the statistical nature of the data. The area under the ROC curve serves as a well-established index of diagnostic accuracy [20]; the minimum value (0.5) arises from assignment by pure chance whereas the maximum value (1.0) corresponds to perfect assignment. ROC calculations permit 

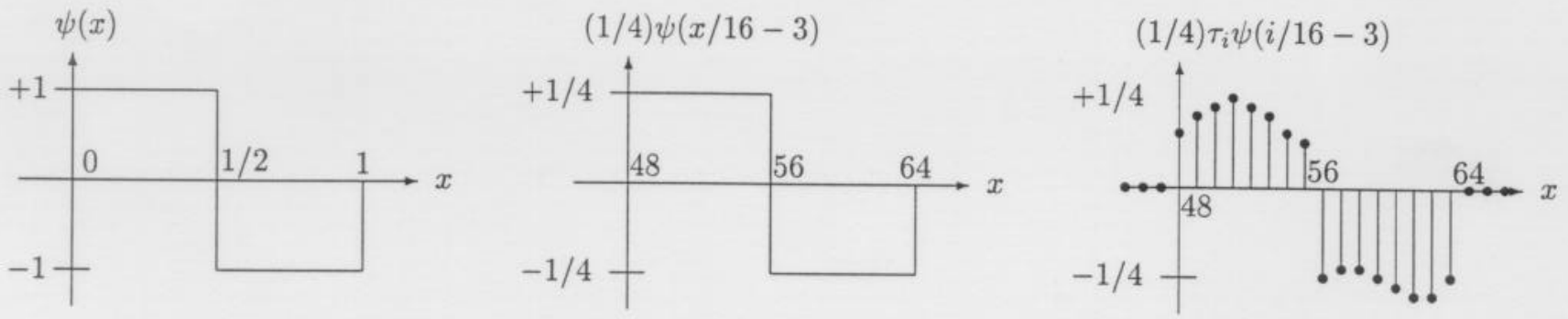

Figure 1: Example of the estimation of a wavelet coefficient using the Haar wavelet. LEFT: Original Haar wavelet. MIDDLE: Delayed and scaled version of the wavelet. RIGHT: Time series multiplied by the wavelet.
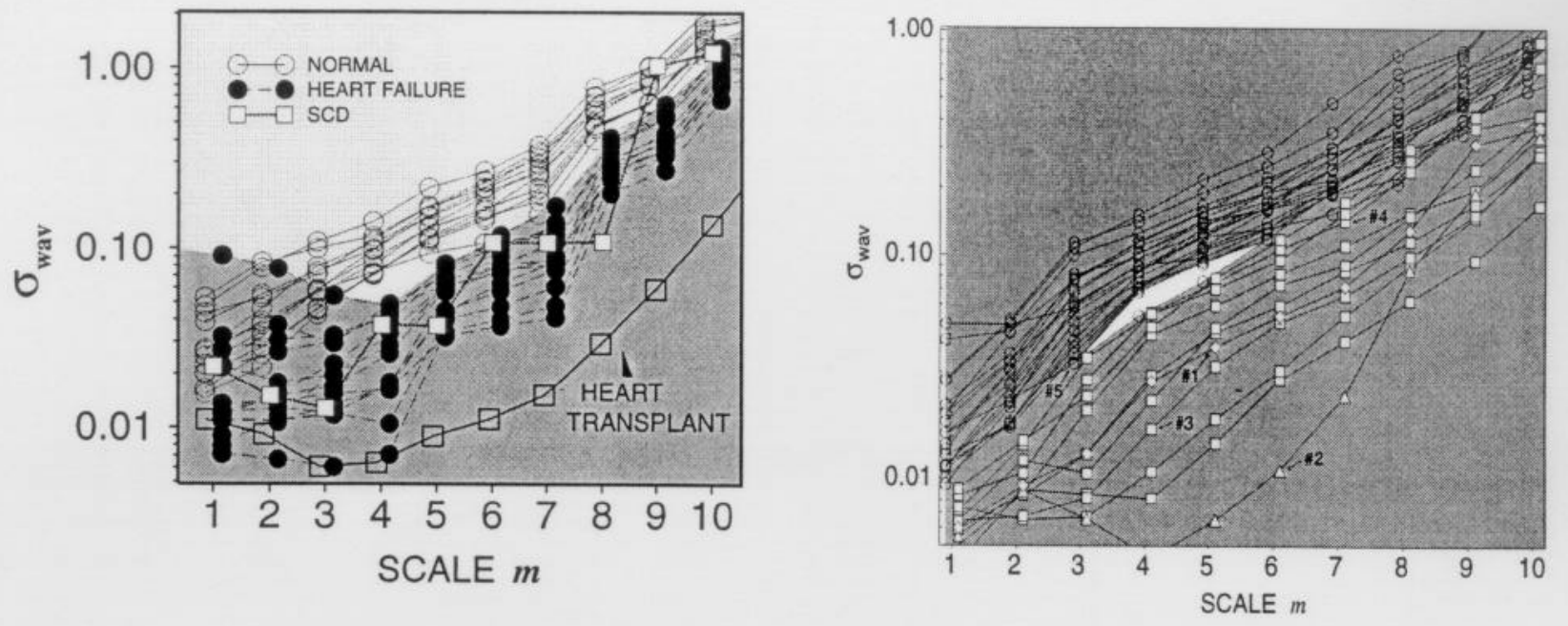

Figure 2: LEFT: Wavelet-coefficient standard deviation $\sigma_{\text {wav }}$ versus scale $m$ for our preliminary study (12 normal subjects indicated by grey circles; 15 heart-failure patients indicated by black circles, one SCD patient indicated by white squares, and one heart-transplant patient indicated by grey squares). RIGHT: $\sigma_{\text {wav }}$ for an Israeli-Danish study conducted by Ashkenazy et al. [14] (21 normal subjects indicated by grey circles; 10 diabetic patients indicated by white squares, one myocardial-infarction patient indicated by white diamonds, and one heart-transplant patient indicated by white triangles). In both studies the best separation of the two groups is achieved at scales 4 and 5 , corresponding to $2^{4}-2^{5}$ heartbeats, despite the differing pathologies and wavelets bases. For the two heart-transplant patients shown, $\sigma_{\text {wav }}$ is severely depressed over a substantial range of scales. 
a quantitative comparison to be made of the abilities of different measures to diagnose the presence of disease.

In Fig. 3 we present the ROC area, as a function of data length (number of beats), using the three different measures. The solid curve in Fig. 3(a) shows ROC area when discriminability is determined by the wavelet-coefficient standard deviation $\sigma_{\text {wav }}(m=5)$ given in Eq. (2). Figure 3 (b) represents the area when the interbeat-interval standard deviation $\sigma_{\text {int }}$ is used instead. The importance of this measure has long been known [22] and it is now commonly used in heart rate variability analysis [23]. Figure 3(c) provides the ROC area for yet another well-known measure, the spectral scaling exponent $\delta$ estimated at ultralow $(<0.003 \mathrm{~Hz})$ and at very-low $(<0.04 \mathrm{~Hz})$ frequencies $[2,23]$.

It is clear from Fig. 3 that $\sigma_{\text {wav }}(m=5)$ is the only measure of the three that, for the ECG recordings in our study, ever achieves an ROC area of unity, thereby indicating perfect ability to separate the heart-failure patients from the normal subjects, and it does so with as few as 20000 heartbeats (corresponding to 4 or 5 hours of data). It is equally evident from Fig. 3 that the measure of choice for ECG recordings with fewer than 3500 heartbeats (corresponding to about 45 minutes of data) is not $\sigma_{\text {wav }}(m=5)$ but rather $\sigma_{\text {int }}$. This transition occurs because $\sigma_{\text {int }}$ depends only on the short-term behavior of the $R-R$ sequence $[12,16]$ whereas the wavelet measure depends on both the short- and long-term behavior.

It is also apparent from Fig. 3 that $\sigma_{\text {wav }}(m=5)$ and $\sigma_{\text {int }}$ always outperform the scaling exponent $\delta$, whatever the data length. Moreover, because $\delta$ reflects the long-duration properties of the interbeat-interval sequence $[12,16]$, its error brackets become unacceptably large as data length decreases [see Fig. 3(c)] so that it can only be reliably calculated for long data sets. Since the scaling parameter introduced by Peng et al. in 1993 [18] is monotonically related to $\delta$ by $2-\delta[16]$, and since the R$R$ recordings were identical in that study and in this, the ROC curve for their scaling parameter is in fact identical to that for $\delta$ given in Fig. 3(c). More generally, scaling exponents derived from the R-R sequence, the spectrum of the generalized heart rate, and the Allan factor (denoted $\delta, \beta$, and $\gamma$, respectively, in [16]) are all essentially the same [16]; this is also expected for the scaling exponent $\alpha$ derived from the wavelet-coefficient standard deviation [24].

We therefore expect that the use of scale-dependent -measures, such as $\sigma_{\text {wav }}(m=5)$ and $\sigma_{\text {int }}$, is likely to prove superior to the use of scale-independent measures such as $\delta, \beta, \gamma, \alpha$, as well as those derived from detrended fluctuation analysis [19] and other related methods.

\section{GENERATING A MATHEMATICAL HEARTBEAT}

The generation of a mathematical point process that faithfully emulates the human heartbeat has importance in a number of venues, including application to pacemaker excitation. Integrate-and-fire (IF) models, which are physiologically plausible, are well-known in cardiology $[25,26]$. In the paper by Berger et al. [26], for example, an integrate-and-fire model was constructed by integrating an underlying rate function $R(t)$ until it reached a fixed threshold $\theta$, whereupon a point event was triggered and the integrator reset. The occurrence time for the $(k+1)$ st beat is then implicitly given by $\theta=\int_{t_{k}}^{t_{k+1}} R(\tau) d \tau$. Modeling the stochastic component of the rate function as bandlimited fractal Gaussian noise (FGN), which introduces scaling behavior into the heart rate, and setting $\theta=1$, results in improved agreement with experiment [16]. This fractal-Gaussian-noise integrate-and-fire (FGNIF) process requires four parameters: the scaling exponent, the relative strength of the FGN spectrum, and lower and upper limits for the noise band. The FGNIF has been quite successful in fitting a whole host of interval- and count-based measures of the heartbeat sequence for both heart-failure patients and normal subjects [16]. It could not, however, accommodate the differences observed in the behavior of $\sigma_{\text {wav }}(m)$ for the two classes of data [21].

To remedy that defect, we constructed a jittered version of this model which we called the FGNJIF $[21,27]$. The process is generated as follows. Preliminary event occurrence times $t_{i}^{\text {pri }}$ are generated by the FGNIF; a Gaussian jitter distribution of standard deviation $J$ is then convolved with each of the $t_{i}^{\mathrm{pri}}$ to determine the times of the final points $t_{i}$ [27]. Increasing the jitter parameter imparts additional randomness to the $\mathrm{R}-\mathrm{R}$ time series at small scales, thereby increasing $\sigma_{\text {wav }}$ at small values of $m$ and, concomitantly, the power spectral density at large values of $f$.

Simulations for the wavelet-coefficient standard deviation $\sigma_{\text {wav }}^{\text {sim }}$ versus scale $m$ using the FGNJIF model have been carried out (see Fig. 2 in [21]). For $J=0$, the results reduce to those for the FGNIF model, and the shape of the simulated curves is in reasonably good accord with experimentally observed curves for normal subjects. As the jitter standard deviation $J$ increases above 0 , the curves bend upward at small values of the scale $m$, and begin to match those for heart-failure patients. The increased jitter also gives rise to a whitening of the spectrum at high frequencies, as expected, so that the distinctions in the spectra between heart-failure patients and normal subjects are properly mimicked by the FGNJIF model (see Fig. 3 in [21]). Typical values of $J$ that accommodate the data lie in the range 0.01 to 0.06 for heart-failure patients and in the range 0 to 0.02 for normal subjects [21]. Values of this 
NORMALIZED AREA UNDER THE ROC CURVES

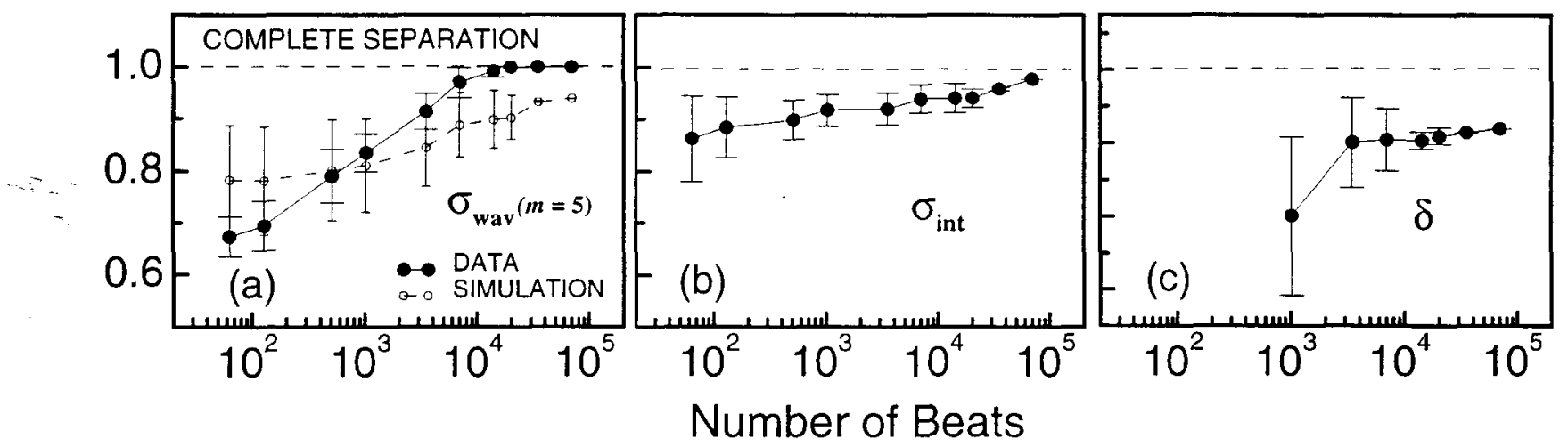

Figure 3: Diagnostic accuracy (area under ROC curve) vs data length (number of heartbeats). A maximum area of unity corresponds to the correct assignment of each patient to the appropriate class. The solid curve in the left panel (a) is obtained by using the wavelet coefficient standard deviation at scale 5 (similar results are obtained at scale 4); the middle panel (b) arises from using the interbeat-interval standard deviation; and the right panel (c) emerges when using the spectral scaling exponent. The areas are based on averages of the first 10 data segments for $64,128,512$, - 1024, 3500, and 7000 events (the leftmost six data points in (a) and (b)), and on 5, 3, 2, and 1 segments of 14000 , 20000,35000 , and 70000 events, respectively (the rightmost 4 data points). $\sigma_{\text {wav }}$ is the only measure of the three that achieves $100 \%$ sensitivity at $100 \%$ specificity for the data in our study, and it does so with as few as 20000 heartbeats (corresponding to 4 or 5 hours of data). For data lengths less than 3500 events (corresponding to about 45 minutes of data), the best performance is provided by $\sigma_{\text {int }}$. The dashed curve in (a) is derived from 27 simulations of the fractal-Gaussian-noise jittered integrate-and-fire (FGNJIF) model (see text).

magnitude result in only a modest (approximately $12 \%$ ) increase in the value of $\sigma_{\text {int }}$ (for comparison, the mean value of $\sigma_{\text {int }}$ for normal subjects is more than twice as large as it is for heart-failure patients [16]).

The FGNJIF simulation does a rather remarkable job of reproducing individual data sets for a number of key measures used in heart-rate-variability analysis (see Fig. 3 in [21]). However, the global performance of the fractal-Gaussian-noise jittered integrate-and-fire (FGNJIF) model for the entire collection of data sets, presented as the dashed curve in Fig. 3(a), does not do as well [21]. Though the simulation follows the trend of the data [solid curve in Fig. 3(a)] quite nicely, there is clearly room for improvement. It will be of interest to examine modifications of the model that will serve to bring the simulated ROC curves into better accord with the data-based curves.

\section{References}

[1] J. B. Bassingthwaighte, L. S. Liebovitch, and B. J. West, Fractal Physiology (Oxford Univ. Press, New York, 1994).

[2] M. Kobayashi and T. Musha, " $1 / f$ fluctuation of heartbeat period," IEEE Trans. Biomed. Eng. BME29, 456-457 (1982).
[3] S. Akselrod, D. Gordon, F. A. Ubel, D. C. Shannon, A. C. Barger, and R. J. Cohen, "Power spectrum analysis of heart rate fluctuation: A quantitative probe of beat-to-beat cardiovascular control," Science 213, 220-222 (1981).

[4] I. Daubechies, Ten Lectures on Wavelets (Society for Industrial and Applied Mathematics, Philadelphia, 1992).

[5] S. G. Mallat, "A theory for multiresolution signal decomposition: The wavelet representation," IEEE Trans. Pattern Anal. Mach. Intell. 11, 674-693 (1989).

[6] Y. Meyer, Ondelettes et Opérateurs (Hermann, Paris, 1990).

[7] Wavelets in Medicine and Biology, edited by A. Aldroubi and M. Unser (CRC Press, Boca Raton, FL, 1996).

[8] Time Frequency and Wavelets in Biomedical Signal Processing, edited by M. Akay (IEEE Press, Piscataway, NJ, 1997).

[9] A. Arneodo, G. Grasseau, and M. Holschneider, "Wavelet transform of multifractals," Phys. Rev. Lett. 61, 2281-2284 (1988). 
[10] P. Abry and P. Flandrin, "Point processes, long-range dependence and wavelets," in Wavelets in Medicine and Biology (CRC Press, Boca Raton, FL, 1996), pp. 413-437.

[11] M. C. Teich, C. Heneghan, S. B. Lowen, and R. G. Turcott, "Estimating the fractal exponent of point processes in biological systems using waveletand Fourier-transform methods," in Wavelets in Medicine and Biology (CRC Press, Boca Raton, FL, 1996), pp. 383-412.

[12] S. Thurner, M. C. Feurstein, and M. C. Teich, "Multiresolution wavelet analysis of heartbeat intervals discriminates healthy patients from those with cardiac pathology," Phys. Rev. Lett. 80, 1544-1547 (1998).

[13] The R-R recordings were drawn from the BethIsrael Hospital (Boston, MA) Congestive HeartFailure Database comprising 12 records from normal patients (age: 29-64 years, mean 44 years) and 15 records from severe congestive heart-failure patients (age 22-71 years, mean 56 years). The recordings, which form a standard database for evaluating the merits of various measures for identifying heart failure, were made with a Holter monitor, digitized at a fixed value of $250 \mathrm{~Hz}$. Three of the 15 heart-failure patients also suffered from atrial fibrillation. Detailed characterization of the data sets is presented in Table 1 of Ref. [16]. We used the first $M=70000$ interbeat intervals (total time duration $T \approx 20 \mathrm{~h}$ ) of each of these records.

[14] - Y. Ashkenazy, M. Lewkowicz, J. Levitan, H. Moelgaard, P. E. Bloch Thomsen, and K. Saermark, "Discrimination of the healthy and sick cardiac autonomic nervous system by a new wavelet analysis of heartbeat intervals," Fractals 6, in press (1998) [Medical Physics e-print physics/9804030;

$\therefore$.http://xxx.sissa.it/list/physics.med-ph/recent].

[15] R. G. Turcott and M. C. Teich, "Long-duration correlation and attractor topology of the heartbeat rate. differ for healthy patients and those with heart failure," Proc. SPIE (Chaos in Biology and Medicine) 2036, 22-39 (1993).

[16] R. G. Turcott and M. C. Teich, "Fractal character of the electrocardiogram: Distinguishing heart-failure and normal patients," Ann. Biomed. Eng. 24, 269-293 (1996).

-[17] M. C. Teich, "Fractal behavior of the electrocardiogram: Distinguishing heart-failure and normal patients using wavelet analysis," Proc. Int. Conf. IEEE Eng. Med. Biol. Soc. 18, 1128-1129 (1996).
[18] C.-K. Peng, J. Mietus, J. M. Hausdorff, S. Havlin, H. E. Stanley, and A. L. Goldberger, "Long-range anticorrelations and non-Gaussian behavior of the heartbeat," Phys. Rev. Lett. 70, 1343-1346 (1993).

[19] C.-K. Peng, S. Havlin, H. E. Stanley, and A. L. Goldberger, "Quantification of scaling exponents and crossover phenomena in nonstationary heartbeat time series," Chaos 5, 82-87 (1995).

[20] J. A. Swets, "Measuring the accuracy of diagnostic systems," Science 240, 1285-1293 (1988).

[21] S. Thurner, M. C. Feurstein, and M. C. Teich, "ROC analysis and a realistic model of heart rate variability," submitted for publication.

[22] M. M. Wolf, G. A. Varigos, D. Hunt, and J. G. Sloman, "Sinus arrhythmia in acute myocardial infarction," Med. J. Australia 2, 52-53 (1978).

[23] M. Malik, J. T. Bigger, A. J. Camm, R. E. Kleiger, A. Malliani, A. J. Moss, and P. J. Schwartz, "Heart rate variability: Standards of measurement, physiological interpretation, and clinical use," Euro. Heart J. 17, 354-381 (1996).

[24] In the region of large $m$, the scaling exponent $\alpha$ is estimated from an individual wavelet-coefficient standard-deviation curve (as shown, for example, in Fig. 2) by calculating the slope of its square (rendering it a variance so that it corresponds to other standard scaling-exponent measures) on a base- $10 \log -\log$ plot. Thus $\alpha=\left[d\left(\log _{10} \sigma_{\text {wav }}^{2}(m)\right)\right] /\left[d\left(\log _{10} 2^{m}\right)\right]=$ $\left[2 / \log _{10} 2\right]\left[d\left(\log _{10} \sigma_{\text {wav }}(m)\right) / d m\right]$ $6.644\left[d\left(\log _{10} \sigma_{\text {wav }}(m)\right) / d m\right]$.

[25] B. W. Hyndman and R. K. Mohn, "A model of the cardiac pacemaker and its use in decoding the information content of cardiac intervals," Automedica 1, 239-252 (1975).

[26] R. D. Berger, S. Askelrod, D. Gordon, and R. J. Cohen, "An efficient algorithm for spectral analysis of heart rate variability," IEEE Trans. Biomed. Eng. BME-33, 900-904 (1986).

[27] S. Thurner, S. B. Lowen, M. C. Feurstein, C. Heneghan, H. G. Feichtinger, and M. C. Teich, "Analysis, synthesis, and estimation of fractalrate stochastic point processes," Fractals 5, 565-595 (1997). 